\title{
SPECTRA OF MAGNETIC FLUCTUATIONS AND RELATIVISTIC PARTICLES PRODUCED BY A NONRESONANT WAVE INSTABILITY IN SUPERNOVA REMNANT SHOCKS
}

\author{
Andrey E. Vladimirov ${ }^{1}$, Andrei M. ByKov ${ }^{2}$, and Donald C. Ellison ${ }^{1}$ \\ ${ }^{1}$ North Carolina State University, Department of Physics, Raleigh, NC 27695-8202, USA; avladim@ncsu.edu, don_ellison@ncsu.edu \\ ${ }^{2}$ Ioffe Physical-Technical Institute, St. Petersburg, Russia; byk@astro.ioffe.ru \\ Received 2009 June 5; accepted 2009 August 17; published 2009 August 31
}

\begin{abstract}
We model strong forward shocks in young supernova remnants with efficient particle acceleration where a nonresonant instability driven by the cosmic ray current amplifies magnetic turbulence in the shock precursor. Particle injection, magnetic field amplification (MFA), and the nonlinear feedback of particles and fields on the bulk flow are derived consistently. The shock structure depends critically on the efficiency of turbulence cascading. If cascading is suppressed, MFA is strong, the shock precursor is stratified, and the turbulence spectrum contains several discrete peaks. These peaks, as well as the amount of MFA, should influence synchrotron X-rays, allowing observational tests of cascading and other assumptions intrinsic to the nonlinear model of nonresonant wave growth.
\end{abstract}

Key words: acceleration of particles - cosmic rays - instabilities - magnetic fields - shock waves - supernova remnants

\section{INTRODUCTION}

Diffusive shock acceleration (DSA) in supernova remnant (SNR) shocks is the most likely mechanism for the origin of the bulk of galactic cosmic rays (CRs) up to at least $10^{15} \mathrm{eV}$. To produce the observed density of galactic CRs, the acceleration mechanism must be efficient and, therefore, nonlinear (e.g., Blandford \& Eichler 1987; Malkov \& Drury 2001; Hillas 2005). X-ray imaging of young SNRs reveals thin synchrotron filaments that are best explained as originating from shock accelerated $\mathrm{TeV}$ electrons radiating in strongly amplified circumstellar magnetic fields (see Vink \& Laming 2003; Bamba et al. 2003; Uchiyama et al. 2007; Vink 2008, and references therein). These fields are almost certainly produced as part of the DSA process (e.g., Bell \& Lucek 2001; Bell 2004; Amato \& Blasi 2006; Pelletier et al. 2006; Vladimirov et al. 2006; Zirakashvili \& Ptuskin 2008; Malkov \& Diamond 2009; Riquelme \& Spitkovsky 2009).

Bell (2004) suggested that magnetic fluctuations in a shock precursor may be amplified by a fast, nonresonant instability induced by the strong diffusive electric current of the shockaccelerated CRs. The transverse MHD waves produced by this mechanism have wavelengths much shorter than the wave generating CR gyroradii. In efficient DSA, the CR current driving the instability must be strongly coupled to the turbulent magnetic fields and supersonic MHD flow. To address this issue, we simulated the amplification of Bell's modes as part of a nonlinear Monte Carlo (MC) model of DSA (Vladimirov et al. 2006). We self-consistently model four basic strongly coupled components of the system: the bulk plasma flow, the full particle spectrum, the self-generated MHD turbulence including cascading, and thermal particle injection. The efficiently produced CRs modify the upstream flow speed, and the CR current determines the growth of stochastic magnetic fields. The fields, in turn, set the particle diffusive transport properties and, subsequently, the injection and acceleration efficiency of the particles, closing the system.

\section{THE MODEL}

Our MC model of nonlinear particle acceleration with strong, resonant, MFA is presented in Vladimirov et al. $(2006,2008)$.
Here, we replace resonant growth with the fast nonresonant instability of Bell and include the cascading of that turbulence to shorter wavelengths. The momentum and space dependent particle mean free paths are calculated consistently with the turbulence.

Consider the nonlinear precursor of a strong, plane-parallel, steady-state shock. In the reference frame comoving with the subshock (at $x=0$ ), where the plasma flows in the positive $x$-direction, the flow speed $u(x)$ has the value $u_{0}$ far upstream. The speed, $u(x)$, drops in the precursor until it obtains the downstream speed $u_{2}=u_{0} / R_{\mathrm{tot}}$, where $R_{\mathrm{tot}}$ is the overall shock compression ratio. The nonresonant current instability is assumed to be the source of the wave spectrum energy density $W(x, k)$ (here $k$ is the wavenumber, and $W d k$ is the amount of energy in the interval $d k$ per unit spatial volume) according to the following equation:

$$
u \frac{\partial W}{\partial x}+\frac{\partial \Pi}{\partial k}=\gamma_{\mathrm{nr}} W-L
$$

The quantity $\Pi(x, k)$ describes cascading, i.e., the transfer of turbulence energy from long to short wavelengths, $L(x, k)$ represents the dissipation of turbulence, and $\gamma_{\mathrm{nr}}(x, k)$ is the quasi-linear rate of wave energy amplification by the instability, and is given by (see Bell 2004)

$$
\gamma_{\mathrm{nr}}=2 v_{A} k \sqrt{\frac{k_{c}}{k}-1}, \quad \text { for } 1 / r_{\mathrm{g} 1}<k<k_{c} .
$$

Here $v_{A}(x)=B_{0} / \sqrt{4 \pi \rho(x)}$ is the Alfvén speed, $c$ is the speed of light, $B_{0}$ is the far upstream magnetic field directed toward the shock normal, $\rho(x)$ is the thermal plasma mass density, $r_{\mathrm{g} 1}(x)$ is the gyroradius of the least energetic current generating CR, the critical wavenumber $k_{c}(x)=4 \pi j_{d}(x) /\left(c B_{0}\right)$, and the local diffusive electric current of CRs responsible for the instability, $j_{d}(x)$, is determined by the MC simulation.

Bell's derivation of (2) assumes that the gyroradii of the streaming CRs are much greater than the wavelengths of the generated waves, which is expressed here by the condition $1 / r_{\mathrm{g} 1}<k$. The growth rate, $\gamma_{\mathrm{nr}}$, has a maximum at $k=k_{c} / 2$ and vanishes for $k>k_{c}$.

Nonlinear interactions between turbulent harmonics may lead to a re-distribution of the energy of turbulent fluctuations in 
$k$-space (i.e., spectral energy transfer). The term $\partial \Pi / \partial k$ in Equation (1), with $\Pi>0$, describes a transfer of energy from large to small turbulent fluctuations (i.e., cascading). Such a description, i.e., the Kolmogorov cascade model, has been successful in explaining the spectra of locally isotropic, incompressible turbulence in non-conducting fluids, observed in experiments and simulations (e.g., Biskamp 2003). However, it is uncertain how spectral transfer operates in a collisionless shock precursor with a CR current strong enough to modify the MHD modes, and in the presence of strong magnetic turbulence. In weak MHD turbulence, cascading was shown to be anisotropic (Goldreich \& Sridhar 1995): harmonics with wavenumbers transverse to the mean magnetic field experience a Kolmogorov-like cascade, while the cascade in wavenumbers parallel to the mean field is suppressed. Diamond \& Malkov (2007) argue for an inverse cascade (i.e., from short to long wavelengths) of turbulent harmonics in the context of magnetic field amplification in DSA. Spectral energy transfer in the form of anisotropic diffusion of energy in $k$-space was proposed by Matthaeus et al. (2009). MHD modeling of Bell's instability with fixed CR current, performed by Bell (2005) and Zirakashvili et al. (2008), show that the magnetic structures consist of expanding walls of strong magnetic field spiraling around cavities in density and magnetic field. Moreover, Zirakashvili et al. (2008) revealed that the turbulent energy is nonlinearly transferred to both longer and shorter scales (see their Figure 4). It should be noted, however, that the MHD models ignored both the interaction of $\mathrm{CR}$ particles with the instability-generated turbulence, and the feedback of the turbulence on the CR current that are the main subjects of our study. The deflection of CRs by magnetic fluctuations may lead to important nonlinear effects, such as a saturation of the instability (e.g., Bell 2005; Pelletier et al. 2006). A complete numerical description of turbulence, which includes the evolution of the fields and the motion of the particles, with a realistic dynamic range, is currently unfeasible. Because the primary goal of our present research is to study the nonlinear aspects of DSA with Bell's instability including the interaction of CR particles with the instability-generated turbulence, approximations must be made for the poorly known details of the turbulent spectral energy transfer. In this work, we investigate the consequences of different spectral energy transfer regimes by presenting two limiting cases. In one (shown with solid curves in all plots), cascading is fully suppressed, i.e., $\Pi=0$. In the other (shown with dotted curves in all plots), the cascading from large to small scales is efficient and has the differential form (see, e.g., Verma 2004), $\Pi=W^{3 / 2} k^{5 / 2} \rho^{-1 / 2}$, corresponding to Kolmogorov's model. ${ }^{3}$

The dissipation term, $L$, is assumed to be zero with no cascading and to have the form of viscous dissipation, $L=$ $v_{A} k^{2} k_{d}^{-1} W$, in the model with cascading (e.g., Vainshtein et al. 1993). The wavenumber, $k_{d}$, is identified with the inverse of a thermal proton gyroradius: $k_{d}(x)=e B_{0} /\left[c \sqrt{m_{p} k_{B} T(x)}\right]$, where $m_{p}$ is the proton mass, $k_{B}$ is Boltzmann's constant, and $T(x)$ is the local gas temperature determined from the heating induced by $L$, as described in Vladimirov et al. (2008). We will consider elsewhere models of spectral transfer different from Kolmogorov cascading.

To highlight the effects of the Bell nonresonant instability and cascading in strong SNR shocks of SNRs, we here ignore resonant instabilities and the compression of turbulence (see

\footnotetext{
3 Note that we ignore a constant on the order of unity in this expression (e.g., Verma 2004).
}

Vladimirov et al. 2006), even though these effects may play an important role in weaker shocks (see Pelletier et al. 2006). We have performed more complete calculations including resonant and nonresonant instabilities with compression and cascading (to be demonstrated elsewhere) and confirmed that the qualitatively new findings presented here stand out even when resonant instabilities and compression are included.

Equation (1) is integrated with respect to $x$ using the far upstream $(x=-\infty)$ boundary condition $W \propto k^{-1}$. We assume that these far upstream seed fluctuations are linear waves that are not subject to cascading or dissipation, and that the transition to the turbulent regime takes place at a position $x_{t}$ where the amplified wave spectrum reaches the value $k W\left(x_{t}, k\right)=B_{0}^{2} / 4 \pi$ at some $k$. At this point, in the model with cascading, $\Pi$ and $L$ are set from zero to the values defined above.

The mean free path of a particle with momentum $p, \lambda(x, p)$, is determined by the MHD turbulence. We calculate $\lambda$ by combining well known theories in different parameter ranges. For the highest energy particles with gyroradii, $r_{g}$, that exceed the turbulence correlation length $l_{\text {cor }}$, we assume $\lambda=r_{g}^{2} / l_{\text {cor }} \propto$ $p^{2}$, as described in Toptygin (1985; see also Jokipii 1971). Particles with $r_{g}$ that resonate with the field fluctuations are assumed to have $\lambda=r_{g} / \mathcal{F}$ (e.g., Blandford \& Eichler 1987), where $\mathcal{F}$ is the normalized product $k W$ evaluated at $k$ resonant with the particle momentum $p$. The lowest energy particles, with $r_{g} \ll l_{\text {cor }}$, are assumed to be trapped by turbulent vortices, and their diffusion is determined by the dynamics of these vortices; these particles are assumed to have $\lambda=l_{\text {cor }}$, independent of $p$ (e.g., Palmer 1982). Our model for $\lambda$ smoothly interpolates between these regimes by introducing a wavenumber $k_{*}$ that, for a given particle momentum $p$, separates the turbulence spectrum $W$ into the small-scale $\left(k>k_{*}\right)$ and the large-scale $\left(k<k_{*}\right)$ parts. In addition, we assume that thermal particles not yet injected into the acceleration process have the Bohm mean free path $\lambda=r_{g}$. Full details of the model are given in Vladimirov (2009).

The diffusive CR current $j_{d}(x)$ is calculated as the corresponding moment of the accelerated proton distribution function $f(x, \mathbf{p})$ derived by the MC simulation.

At every upstream location $x$, the minimal CR momentum $p_{1}$ is defined from the particle distribution function, $f(x, \mathbf{p})$, as the momentum below which the CRs contribute $1 \%$ of the total CR pressure. Then $p_{1}$ is used to calculate the gyroradius $r_{\mathrm{g} 1}$ that determines the applicability range of Equation (2). We assume $\gamma_{\mathrm{nr}}=0$ outside of that range of $k$.

\section{RESULTS}

For both examples shown here, we model a shock of speed $u_{0}=10^{4} \mathrm{~km} \mathrm{~s}^{-1}$ propagating in a uniform magnetic field $B_{0}=$ $3 \mu \mathrm{G}$ in a plasma with proton density $n_{0}=0.3 \mathrm{~cm}^{-3}$ and temperature $T_{0}=10^{4} \mathrm{~K}$. We assumed that the acceleration process is in steady-state and size-limited with a free escape boundary (FEB) at $x_{\mathrm{feb}}=-10^{7} r_{g 0}$, where $r_{g 0} \equiv m u_{0} c / e B_{0} \approx 3.5 \times 10^{10} \mathrm{~cm}$.

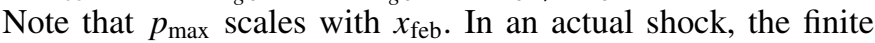
size, and/or finite age, of the shock will determine $p_{\max }$.

In Figure 1 we compare the flow speed, $u(x)$, the amplified effective field, $B_{\text {eff }}(x)$, the CR current, $j_{d}(x)$, and the thermal plasma temperature, $T(x)$, for the two models. With no cascading (solid curves), the overall compression ratio $R_{\mathrm{tot}}=$ $u_{0} / u_{2} \approx 15$, and the downstream field, $B_{\text {eff }}(x>0) \approx 1000 \mu \mathrm{G}$. These quantities are substantially reduced with cascading (dotted curves), i.e., $R_{\text {tot }} \approx 11$, and $B_{\text {eff }}(x>0) \approx 100 \mu \mathrm{G}$. 


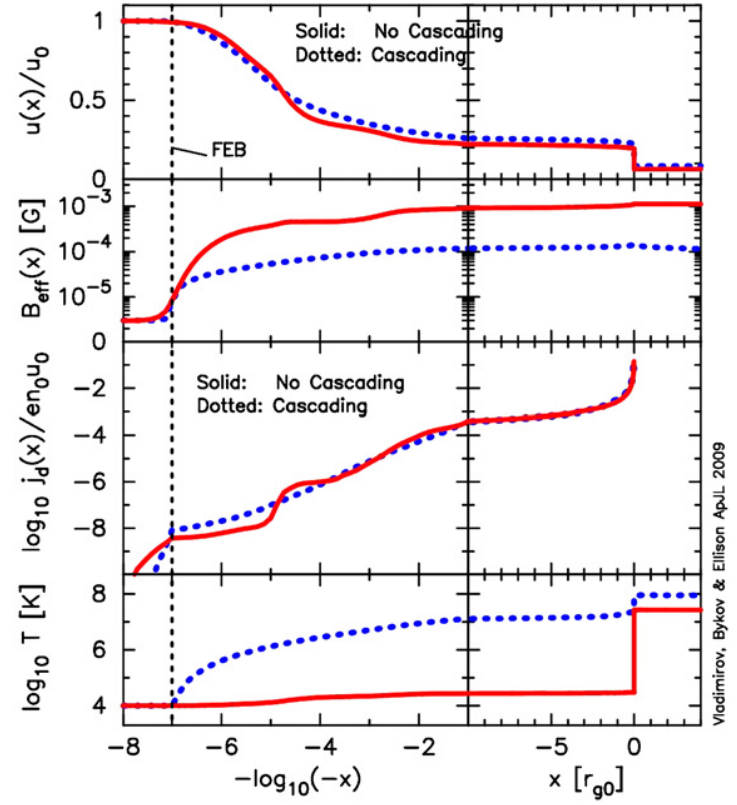

Figure 1. Flow speed $u$, effective magnetic field $B_{\text {eff }}$, diffusive CR current $j_{d}$, and temperature $T$ vs. position $x$. Solid curves are without cascading and dotted curves include strong Kolmogorov cascading. The viscous subshock is at $x=0$ and the position of the FEB is indicated.

There are also differences in $j_{d}(x)$, where $j_{d}$ is smooth with cascading but shows an uneven structure without cascading. The parameter that determines the scale of the generated turbulence, $k_{c}$, has the same $x$-dependence as $j_{d}$. This uneven precursor structure has a striking effect on the magnetic turbulence (see Figure 3). Another prominent difference is the significantly increased precursor temperature, $T(x)$, with cascading due to the dissipation of turbulence at large $k$ (see Vladimirov et al. 2008).

In Figure 2 we plot the downstream $\left(x=5 r_{g 0}\right)$ particle distribution $p^{4} f(x, p)$ and mean free path $\lambda(x, p) / r_{g 0}$. The maximum momentun, $p_{\max }$, is clearly greater without cascading $\left(\sim 10^{5}\right.$ versus $\left.\sim 2 \times 10^{4} m_{p} c\right)$ but the concave shape for $p^{4} f$ is present in both cases, indicating that both are efficient particle accelerators (e.g., Berezhko \& Ellison 1999). As indicated by the thermal peaks, the shocked temperature is considerably higher in the model with cascading, an effect similar to what was observed in Vladimirov et al. (2008). With cascading (dotted curves), $\lambda$ is a smooth function of $p$ showing the three regions discussed above: $\lambda \sim$ constant for low $p, \lambda \propto p$ for intermediate values of $p$, and $\lambda \propto p^{2}$ for the highest momenta. Without cascading, $\lambda$ is always less than with cascading and shows plateau regions where particles are trapped by vortices of different scales.

\section{DISCUSSION}

Our most intriguing result is shown in Figure 3 where we plot $k W(x, k)$ at different locations, $x$, relative to the subshock, as indicated. The seed turbulence from the unshocked interstellar medium is at the level of the horizontal lines.

Without cascading (solid curves), we see fairly narrow peaks centered at distinct $k$. This is in stark contrast to the case with cascading (dotted curves), and to the broad spectrum of $k W(x, k)$ seen previously with resonant interactions (e.g., Vladimirov et al. 2006). Only the most energetic CRs reach a far upstream position near $x_{\mathrm{feb}}$, and the small current these CRs produce gives a maximum $\gamma_{\mathrm{nr}}$ at small $k=k_{c} / 2$. At $x_{\mathrm{feb}}=-10^{7} r_{g 0}$, where the light-weight curves are calculated

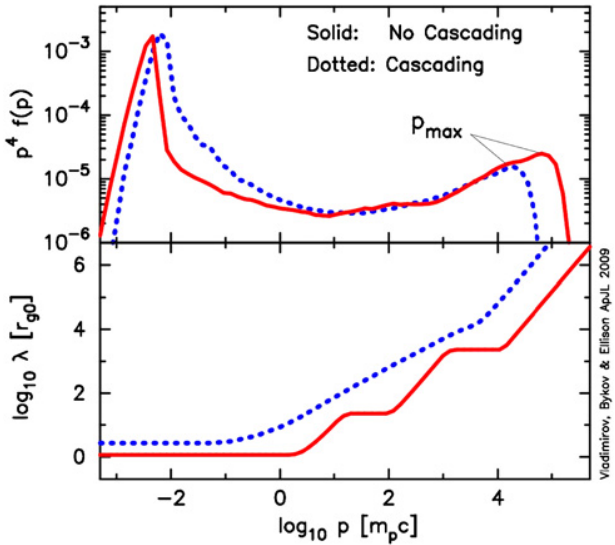

Figure 2. Proton distribution function, $f$, times $p^{4}$, and accelerated particle mean free path, $\lambda$, both calculated downstream of the subshock. The turnover momenta, $p_{\max }$, for the two cascading models are indicated.

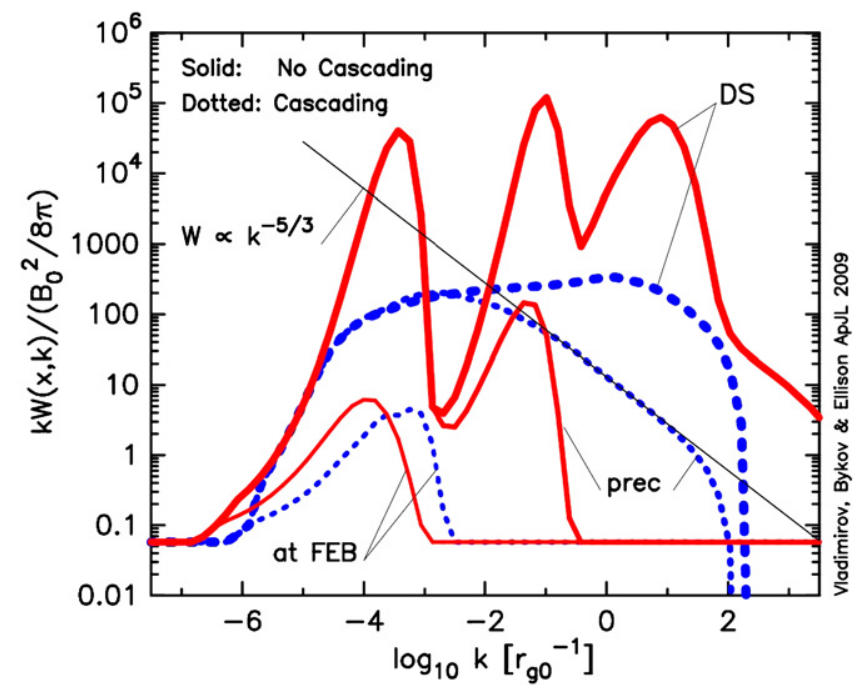

Figure 3. Turbulence spectra, $W$, times $k$, at different locations relative to the subshock. The lightest-weight curves are calculated at $x_{\mathrm{feb}}=-10^{7} r_{g 0}$, the heaviest curves are calculated in the downstream region, and the medium-weight curves are calculated in the precursor at $x=-1.2 \times 10^{4} r_{g 0}$. The far upstream seed turbulence (at all $k$ ) is at the level indicated by the horizontal lines.

in Figure 3, $\gamma_{\mathrm{nr}}$ is a maximum at $k \sim 1 \times 10^{-4} r_{g 0}^{-1}$ with no cascading, and at $k \sim 5 \times 10^{-4} r_{g 0}^{-1}$ with cascading. The difference corresponds to the difference in escaping CR flux at $x_{\mathrm{feb}}$. At a position within the precursor at $x=-1.2 \times 10^{4} r_{g 0}$ (medium-weight curves), a second peak at larger $k$ has developed when cascading is suppressed. At this position closer to the subshock, lower energy particles appear in large numbers and the generation of waves at $k \sim 10^{-4} r_{g 0}$ shuts down because of the range in $k$ given in Equation (2). The increased number of lower energy particles, and the accordingly increased $j_{d}$, now corresponds to a greater $k_{c}$ and shorter wavelength structures get amplified at $k \sim 10^{-1} r_{g 0}^{-1}$. The position of the leftmost peak is nearly constant between $x_{\mathrm{feb}}$ and $-1 \times 10^{5} r_{g 0}$ because $j_{d}$ is nearly constant in this range, as indicated in Figure 1. At the downstream position (heaviest solid curve), three distinct peaks are generated without cascading.

The same turbulence generation model with cascading shifts energy from small to large $k$ and produces smooth spectra. Far enough upstream in the precursor but not at $x_{\text {feb }}$ (mediumweight dotted curve), where only relatively long wavelengths are amplified, the spectrum exhibits the "injection range" at 
$k \lesssim 10^{-3} r_{g 0}^{-1}$, the broad "inertial range" $10^{-3} r_{g 0}^{-1}<k<$ $10 r_{g 0}^{-1}$ with the Kolmogorov spectrum $W \propto k^{-5 / 3}$, and the "dissipation range" at $k \gtrsim 10 r_{g 0}$. Closer to the subshock, the increased $j_{d}$ shifts turbulence amplification to greater $k$ making the spectrum harder. The downstream spectrum becomes close to $W \propto k^{-1}$ over a broad range in $k$.

The peaks occur because of the coupling of particle transport with turbulence amplification. The first (smallest $k$ ) peak forms far upstream, where only the highest energy particles are present, and their current $j_{d}$ is low. These particles diffuse in the $\lambda \propto p^{2}$ regime, scattered by the short-scale magnetic field fluctuations that they themselves generate. As the plasma moves toward the subshock, advecting the turbulence with it, lower energy particles appear. At some $x$, particles with energies low enough to resonate with the turbulence generated farther upstream (in the lowest $k$ peak) become dominant. This strong resonant scattering leads to a high gradient of $j_{d}$ (seen at $x \sim-10^{5} r_{g 0}$ in the third panel of Figure 1), and the wavenumber $k_{c} / 2$, at which the amplification rate $\gamma_{\mathrm{nr}}$ has a maximum, increases rapidly. The increased value of $k_{c} / 2$ leads to the emergence of the second peak between $10^{-2}$ and $10^{-1} r_{g 0}^{-1}$, as seen in Figure 3. Similarly, the third peak is generated at distances closer to the subshock than $\sim-1 \times 10^{4} r_{g 0}$ and this is seen in the heavy-weight, downstream spectrum in Figure 3 at $k \sim 10 r_{g 0}^{-1 .}$.

The number of peaks depends on the dynamic range, i.e., on $x_{\mathrm{feb}}$. A smaller $x_{\mathrm{feb}}$ can result in two peaks, while a larger $x_{\mathrm{feb}}$, and therefore a larger $p_{\max }$, can yield four or more peaks in the downstream region. As mentioned above, $x_{\mathrm{feb}}$ is a parameter in our model and is chosen here to give $E_{\max } \sim 10-100 \mathrm{TeV}$.

The formation of the spectrum with discrete peaks occurs simultaneously with the stratification of the shock precursor into layers (see the plots of $j_{d}$ ), in which vortices of different scales are formed. The peaks are a direct result of Bell's nonresonant instability, but they will not show up unless $\lambda$ and $\gamma_{\mathrm{nr}}$ are calculated consistently, and the simulation has a large enough dynamic range in both $k$ and $p$.

As emphasized above, cascading in strong turbulence is uncertain and our two models bound the extremes of no cascading and Kolmogorov cascading to larger $k$. Our results are also limited by the fact that we have not considered the case of energy transfer from short to long scales. Nevertheless, the stark differences we see in both the shock structure and the turbulence spectrum suggest that observations may be able to constrain cascading models. The stratification is eliminated and the peaks are spread out if rapid Kolmogorov cascading is assumed. Downstream, the spectrum becomes close to, but slightly flatter than, $W \propto k^{-1}$. Furthermore, the amplified effective magnetic field, the shock compression ratio, and the maximum energy of accelerated particles are all significantly smaller with cascading.

The large amount of energy observed in the first (smallest $k$ ) peak of the turbulence spectrum without cascading defines a potentially observable spatial scale. For the parameters used here, $D_{\max } \approx 2 \pi / k \approx 2 \times 10^{4} r_{g 0} \approx 10^{15} \mathrm{~cm}$, but this depends on $x_{\mathrm{feb}}$, and we have found (work in preparation) that $D_{\max } \propto\left|x_{\mathrm{feb}}\right| \propto p_{\max }$. Therefore, spatially resolved intensity variations translate to $D_{\max }$ (see Bykov et al. 2008) and may offer a new way to estimate $p_{\max }$ from $\mathrm{X}$-ray synchrotron observations of SNRs. For our parameters, the turbulence on the scale $D_{\max }$ has the effective magnetic field $\Delta B \approx \sqrt{4 \pi k W} \approx 400 \mu \mathrm{G}$, and the corresponding Alfvén speed $v_{A} \approx 400 \mathrm{~km} \mathrm{~s}^{-1}$, giving a characteristic timescale $t=D_{\max } / v_{A} \approx 0.5 \mathrm{yr}$. A larger $p_{\max }$ implies a larger $t$. This rapidly varying field may influence both the spectral shape and the time variability of X-ray synchrotron emission in SNRs (see, e.g., Uchiyama et al. 2007; Bykov et al. 2008).

On the other hand, if cascading to short scales is important, magnetic energy is efficiently transferred to the background gas and the amplified magnetic field is considerably reduced. The heating of the shock precursor by the dissipated turbulence will also be significant. As shown in the bottom panel of Figure 1, the precursor temperature can be orders of magnitude greater with cascading than without. The low-energy superthermal particles (up to $p \approx m_{p} c$ ) are more abundant in the presence of cascading and dissipation (the top panel of Figure 2) due to heatingboosted injection (Vladimirov et al. 2008), which may influence bremsstrahlung and spectral line emission in SNRs.

We thank the anonymous referee for the constructive and useful comments. We acknowledge support from NASA grants ATP02-0042-0006, NNH04Zss001N-LTSA, 06-ATP06-21, and RBRF grant 09-02-12080.

\section{REFERENCES}

Amato, E., \& Blasi, P. 2006, MNRAS, 371, 1251

Bamba, A., Yamazaki, R., Ueno, M., \& Koyama, K. 2003, ApJ, 589, 827

Bell, A. R. 2004, MNRAS, 353, 550

Bell, A. R. 2005, MNRAS, 358, 181

Bell, A. R., \& Lucek, S. G. 2001, MNRAS, 321, 433

Berezhko, E. G., \& Ellison, D. C. 1999, ApJ, 526, 385

Biskamp, D. 2003, Magnetohydrodynamic Turbulence (Cambridge: Cambridge Univ. Press), 297

Blandford, R., \& Eichler, D. 1987, Phys. Rep., 154, 1

Bykov, A. M., Uvarov, Y. A., \& Ellison, D. C. 2008, ApJ, 689, L133

Diamond, P. H., \& Malkov, M. A. 2007, ApJ, 654, 252

Goldreich, P., \& Sridhar, S. 1995, ApJ, 438, 763

Hillas, A. M. 2005, J. Phys. G: Nucl. Part. Phys., 31, 95

Jokipii, J. R. 1971, Rev. Geophys. Space Phys., 9, 27

Malkov, M. A., \& Diamond, P. H. 2009, ApJ, 692, 1571

Malkov, M. A., \& Drury, L. 2001, Rep. Prog. Phys., 64, 429

Matthaeus, W. H., Oughton, S., \& Zhou, Y. 2009, Phys. Rev. E, 79, 035401

Palmer, I. D. 1982, Rev. Geophys. Space Phys., 20, 335

Pelletier, G., Lemoine, M., \& Marcowith, A. 2006, A\&A, 453, 181

Riquelme, M. A., \& Spitkovsky, A. 2009, ApJ, 694, 626

Toptygin, I. N. 1985, Cosmic Rays in Interplanetary Magnetic Fields (Dordrecht: Reidel)

Uchiyama, Y., Aharonian, F. A., Tanaka, T., Takahashi, T., \& Maeda, Y. 2007, Nature, 449, 576

Vainshtein, S. I., Bykov, A. M., \& Toptygin, I. N. 1993, Turbulence, Current Sheets, and Shocks in Cosmic Plasma (New York: Gordon \& Breach)

Verma, M. K. 2004, Phys. Rep., 401, 229

Vink, J. 2008, AIP Conf. Proc., 1085, 169

Vink, J., \& Laming, J. M. 2003, ApJ, 584, 758

Vladimirov, A., Ellison, D. C., \& Bykov, A. 2006, ApJ, 652, 1246

Vladimirov, A. E. 2009, Ph.D. thesis, NCSU

Vladimirov, A. E., Bykov, A. M., \& Ellison, D. C. 2008, ApJ, 688, 1084

Zirakashvili, V. N., \& Ptuskin, V. S. 2008, ApJ, 678, 939

Zirakashvili, V. N., Ptuskin, V. S., \& Völk, H. J. 2008, ApJ, 678, 255

\footnotetext{
4 The tail at $k>100 r_{g 0}^{-1}$ is produced by the large $j_{d}$ at $-1 r_{g 0}<x<0$ seen in Figure 1. The short-scale turbulence produced by this current contains little power and does not impact our results for larger scales.
} 